Proceedings of the 6th Polish Symposium of Physics in Economy and Social Sciences (FENS2012), Gdańsk, Poland

\title{
Real Estate Market under Catastrophic Change
}

\author{
M. BeŁEJ ${ }^{a, *}$ AND S. KulEsZA ${ }^{b}$ \\ ${ }^{a}$ Department of Real Estate Management and Regional Development, University of Warmia and Mazury in Olsztyn \\ R. Prawocheńskiego 15, 10-720 Olsztyn, Poland \\ ${ }^{b}$ Chair of Relativistic Physics, University of Warmia and Mazury in Olsztyn \\ Słoneczna 54, 10-710 Olsztyn, Poland
}

\begin{abstract}
Within the last decade, real estate prices in Poland have changed significantly. We believe that these prices inevitably reflect the dynamics of the real estate market, and therefore they can be used to track its evolution path. To study whether and how the prices (regarded as a state variable) change over time depending on such control variables as gross domestic product and central bank interest rates, the theory of discontinuous change (also known as the catastrophe theory) was used. Catastrophic model assumes that small price fluctuations are associated with stable, long-term development of the market, whereas rapid changes are always due to short-term instabilities. In such a picture, the system evolution path draws a smooth curve within the stability area passing continuously between neighboring equilibrium states, and it rarely enters into the instability area to jump over the potential barrier to another equilibrium state.
\end{abstract}

DOI: 10.12693/APhysPolA.123.497

PACS: 05.45.-a, 89.20.-a, 89.65.Gh

\section{Introduction}

In many countries, the real estate market represents an important pillar of its national economy. In Poland, following commencement of the systemic transformation of 1990 's, the process of earlier unheard-of real estate market development has been initiated. As a consequence, the prices of residential real estate started to evolve dynamically giving rise to minor long-term fluctuations occasionally perturbed by rapid decreases and increases. The classic methods of modeling the events in the real property market in both the spatial and the time sense are adequate in case of slow changes, however, they possess numerous difficulties in case of unexpected and rapid changes, when the entire market seems to be losing its stability.

As concerns the analyzed issue, the durability of the current trend and the causes for its change represents an intriguing issue. Authors' temporal hypothesis states that occasionally observed sudden changes of real-property prices occur through discontinuous steps caused by searching for the new equilibrium of the overall system. This paper proposes and presents verification of that hypothesis using the mathematic models from the catastrophe theory.

\section{Cusp catastrophe model}

The catastrophe theory has been successfully applied in the studies conducted in many scientific areas on the grounds of both "soft" disciplines, such as philosophy and psychology, and "hard" sciences, such as economics and management, mathematics, biology, geography, chemistry and others [1-8]. In general, all dynamical systems

*corresponding author; e-mail: caprio@uwm.edu.pl continuously tend toward equilibrium associated with the local minima of potential function. The catastrophe theory describes the potential in terms of control variables and state variables. The former determine sensitivity of the system, whereas the latter define its current state.

In particular, the cusp catastrophe theory used throughout this study assumes that the potential $V$ depends on two control variables: asymmetry factor $\alpha$ and bifurcation factor $\beta$, and only one state variable $y$, in the form given by [1]:

$$
V(y, \alpha, \beta)=-\frac{1}{4} y^{4}+\frac{1}{2} \beta y^{2}+\alpha y .
$$

Moreover, each control variable can be actually defined as linear combination of several observables (independent variables):

$$
\begin{aligned}
& \alpha=\alpha_{0}+\sum_{i=1}^{n} \alpha_{i} x_{i}, \\
& \beta=\beta_{0}+\sum_{i=1}^{n} \beta_{i} x_{i} .
\end{aligned}
$$

Equilibrium states of the system are the roots of partial derivative of the potential with respect to the state variable

$$
-\frac{\partial V(y, \alpha, \beta)}{\partial y}=0 .
$$

Assuming that potential is defined as in Eq. (1), all equilibrium solutions form a fold within the state space, similar to that shown in Fig. 1. On the other hand, system in equilibrium does not change with time, and therefore

$$
\frac{\mathrm{d} y}{\mathrm{~d} t}=0 \text {. }
$$

As a result, time-dependent evolution equation of the system takes the form

$$
\mathrm{d} y=-\frac{\partial V(y, \alpha, \beta)}{\partial y} \mathrm{~d} t
$$




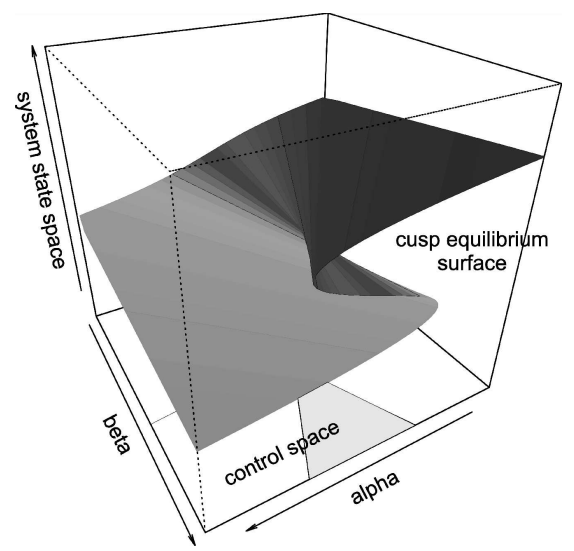

Fig. 1. System equilibrium surface shape in the cusp catastrophe model. The smooth fold related to the change of the system potential shape change and transition from the single equilibrium state (negative values of the bifurcation variable $\beta$ ) to the three-times degenerated equilibrium state (positive values of the bifurcation variable $\beta$ ) is clearly marked within it.

In real world, all dynamical systems evolve in a slightly different manner due to random influence of additional factors. Such systems are called non-deterministic (stochastic), which means that knowing their current state it is not necessarily possible to determine next state. To obtain stochastic form of Eq. (6), Cobb and Watson [9], and Cobb [10] proposed to add Gaussian white noise with a variance $\sigma^{2}$ :

$$
\mathrm{d} y=-\frac{\partial V(y, \alpha, \beta)}{\partial y} \mathrm{~d} t+\sigma(t) \mathrm{d} W
$$

In the above equation, derivative term is referred to as the drift, whereas $W$ is the Wiener process with variance $\sigma^{2}$ $[9,10]$. It is associated with a probability density that describes the distribution of the system's states on any moment in time, which may be expressed as

$$
f(y)=\frac{\Psi}{\sigma^{2}} \exp \left(\frac{-\frac{1}{4} y^{4}+\frac{1}{2} \beta y^{2}+\alpha y}{\sigma^{2}}\right),
$$

where $\Psi$ is a normalizing constant.

Hartelman et al. [11, 12] and Wagenmakers [13] found direct relation between probability density function $f(y)$ associated with Eq. (8) and that corresponding to an asymptotic stationary stochastic process $f_{\mathrm{s}}(y)$. They proved that $f(y)$ and $f_{\mathrm{s}}(y)$ converge over time assuming fast thermalization of the system, that is assuming much faster changes in the system state than in the control variables. Since observables simultaneously contribute to the control variables and affect the state variable, their particular dependence can be determined using the maximum likelihood approach proposed by Cobb and Watson [9], which was further developed by Hartelman [11] and Wagenmakers [13]. This fitting procedure was then implemented in the "cusp" software application used throughout this study, described in detail by Grasman et al. [14].

\section{Catastrophe models in the real estate market}

\subsection{Model variables - state variables and control variables}

As mentioned earlier, potential function in the cusp catastrophe model depends on two control variables: $\alpha$ and $\beta$ that are linearly dependent on chosen observables, which is also true for state variable $y$. The appropriate choice of variables having a robust set of empirical data is of fundamental importance since it constitutes an unhappy compromise between the best possible fit quality and the coherence with basic economic laws. The basic criterion for selection the variables is based on time delay: fluctuations of certain quantities might give rise to destabilization of the market - those will be control variables, while others - changing with some delay will represent balancing the market, so they should be incorporated into state variables.

The main parameter applied in the majority of studies on the real estate market is transaction price (per square meter). To use it as a measure of the system state, transaction prices averaged within monthly intervals need to be calculated. Relevant data were taken from the Register of Prices and Values maintained by the City Administration Office in Olsztyn. Register gathers all types of transactions, both market and non-market ones, and therefore the selection and verification of the entries need to be carried out prior to calculation. All non-market transactions, e.g. deeds of gift or personal easements, were removed. As a result, the number of entries was decreased from 9200 to 5600 of market residential dwelling transactions from May 2003 until December 2010. Based on these data, average unit transaction prices were calculated, and shown in Fig. 2.

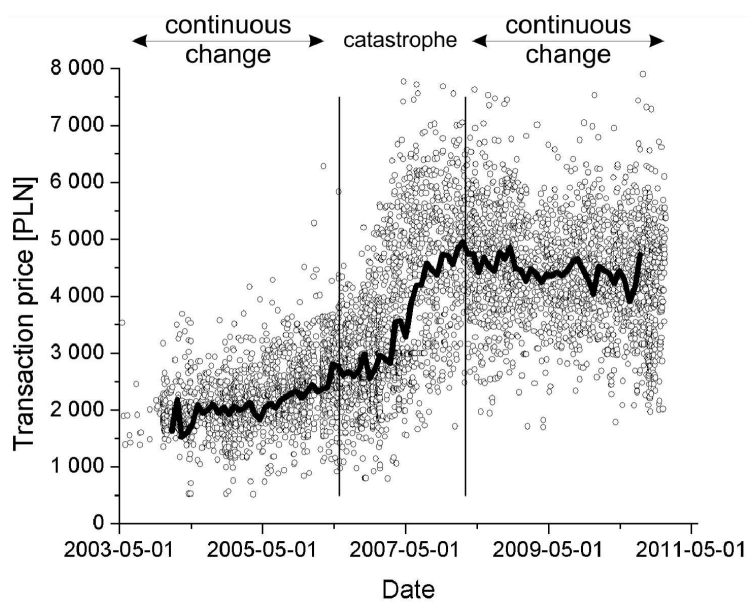

Fig. 2. Real property sale transaction prices in the city of Olsztyn during the period from May 2003 until December 2010 (white points); the continuous line represents the transaction prices averaged at monthly intervals. Source: own work.

As seen in Fig. 2, real property prices slowly increased during the years 2003-2006, then they continued to grow 
much faster between January and December 2007, but after that they gradually fell down (from January 2008 until the end of December 2010). The situation in the first and the third of the above time periods raises no major doubts concerning correlation with cyclical market changes; however, in 2007 we observe a rapid increase in real property prices coupled with a decrease in a number of transactions. The authors suppose it is due to discontinuous change in the real property market structure (morphogenesis), that is qualitative change in market's dynamics when the possibilities of balanced evolution come to an end. To verify such assumption, the histogram presented in Fig. 3 was developed. Figure 3 exhibits two peaks in price distribution (PLN 2000 and PLN 4500) corresponding to two market equilibrium states, which suggests bimodality of the distribution. On the other hand, presence of the repeller (sets of unstable system equilibrium states creating the areas of unavailability), is indicated by the evident decrease in the number of transactions within the price range from PLN 3000 to PLN 4000.

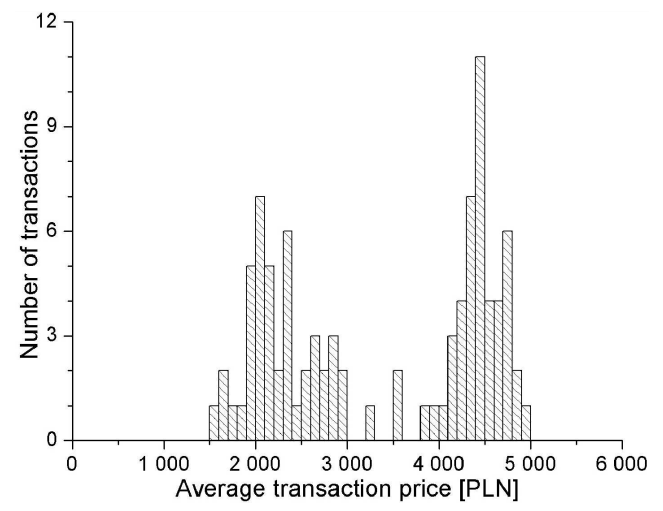

Fig. 3. Histogram of the real property sale transaction prices during the period from June 2003 until December 2010. Source: own work.

Among parameters, which on the ground of various economic theories may potentially influence dynamics of the real property market, one may consider: the central bank interest rates, number and volume of the mortgage loans, currency structure of the loans, exchange rates, unemployment rate, gross domestic product size and changes, economic growth rate, demographic data (population size, age structure, balance of migrations), changes in (un-)employment, household incomes, inflation rate, level of tax burdens for possession of a real property, real property market liquidity and size, infrastructural investments level, hidden economic potential of the real properties (investment attractiveness), costs of construction per square meter of usable area and changes of costs in construction industry $[15,16]$.

Selection of appropriate control variables is particularly important to verify our temporal hypothesis; we understand the need of verification of various parameters and the construction of a number of different models.
However, due to the initial phase of research, set of control variables was finally limited to: quarterly gross domestic product (GDP), averaged reference and lombard rates level (CBIR) and number of transactions (NTR) in monthly intervals. Data regarding the analyzed variables were acquired from the Central Statistical Office (GDP), the National Bank of Poland (CBIR) and the database of property prices in Olsztyn with own calculations (NTR).

\subsection{Results of fitting}

Numerical fitting of the cusp catastrophe models to the available empirical data was conducted making use of the "cuspfit" software package [14]. To assess the validity of the cusp model, additional comparison was made that includes linear and logistic models. The linear model is actually identical to what is called linear subspace regression: the first canonical variate between the independent and dependent variables is used as the predicted value, and the first canonical correlation is used for calculating the explained and residual variance. On the other hand, the logistic model uses non-linear least squares regression to fit canonical data to the logistic curve. To compare the above models, several measures of the fit quality were calculated, namely: pseudo- $R^{2}$, negative logarithmic likeness (logLik), Akaike information criterion (AIC), and Bayesian information criterion (BIC). Two models named, respectively, model $A$ and model $B$ were tested.

In model $A$ (Table I) only two independent variables: the GDP value and the National Bank of Poland interest rates computed as the average of the lombard rate and the reference rate were used. The canonical coordinates were established in the identical way allowing additionally the possibility of the unconstrained fit. The fitting results are presented graphically in Fig. $4 \mathrm{~A}$.
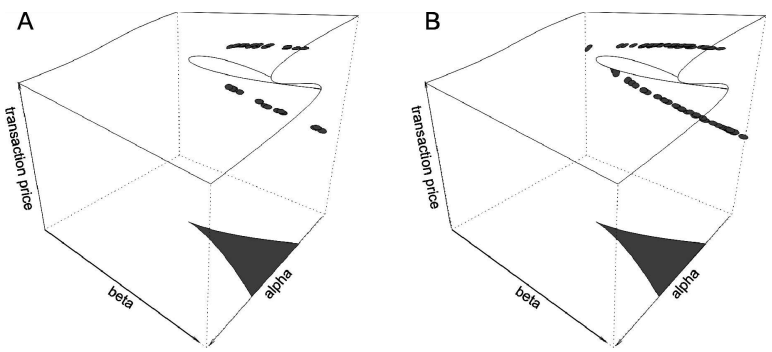

Fig. 4. 3-dimensional visualisation of the empirical data fit to the cusp type catastrophe model. (A) Fit according to model $A$, (B) fit according to model $B$. Almost complete positioning of the state variables (real property transaction prices) within the fold area draws attention as it confirms the catastrophic nature of the phenomena described.

Obtained results indicate that all the observables provide identical contribution to the control parameters. In case of the asymmetry index $\alpha$, the free term (bold data in Table I) proved to be of little significance while in case of the bifurcation index $\beta$ all the variables were found 
significant. This would indicate that the level of interest rates and the volume of generated GDP influenced both the price distribution asymmetry and bifurcation. After modification (free term set to 0 in the asymmetry index (lower part of Table I)), obtained results changed only slightly.

TABLE I

Fit parameters and fit quality indicators for the amended and improved amended model $A$ discussed in the text (GDP - gross domestic product, CBIR - central bank interest rates, NTR - number of transactions. Price - average real estate price per square meter, alpha - asymmetry factor, beta - bifurcation factor, $y$ - state variable). For the comparison purposes the fit indicators for the linear and logistic models are also presented. Values in bold are insignificant from the perspective of the fit quality.

\begin{tabular}{|c|c|c|c|c|c|c|c|}
\hline \multicolumn{8}{|c|}{ Model $A$} \\
\hline \multicolumn{3}{|c|}{ alpha } & \multicolumn{3}{|c|}{ beta } & \multicolumn{2}{|c|}{$y$} \\
\hline GDP & CBIR & Intercept & GDP & CBIR & Intercept & Price & Intercept \\
\hline-0.02305 & -0.2162 & 3.654 & -0.3042 & 1.298 & 28.57 & 0.001658 & -5.582 \\
\hline \multicolumn{4}{|c|}{ fitting quality } & $\overline{R^{2}}$ & logLik & $\mathrm{AIC}$ & $\mathrm{BIC}$ \\
\hline \multicolumn{4}{|c|}{ linear model } & 0.1438666 & -761.40483 & 1530.8097 & 1540.853 \\
\hline \multicolumn{4}{|c|}{ logistic model } & 0.5407698 & -733.06403 & 1480.1281 & 1497.704 \\
\hline \multicolumn{4}{|c|}{ cusp model } & 0.9451956 & -49.24058 & 114.4812 & 134.568 \\
\hline \multicolumn{8}{|c|}{ Improved model $A$} \\
\hline \multicolumn{3}{|c|}{ alpha } & \multicolumn{3}{|c|}{ beta } & \multicolumn{2}{|c|}{$y$} \\
\hline GDP & CBIR & Intercept & GDP & CBIR & Intercept & Price & Intercept \\
\hline 0.01227 & -0.2231 & 0 & -0.3133 & 1.294 & 29.56 & 0.001659 & -5.582 \\
\hline \multicolumn{4}{|c|}{ fitting quality } & $R^{2}$ & $\log \mathrm{Lik}$ & AIC & $\mathrm{BIC}$ \\
\hline \multicolumn{4}{|c|}{ linear model } & 0.1438666 & -761.40483 & 1530.8097 & 1540.8531 \\
\hline \multirow{2}{*}{\multicolumn{4}{|c|}{ logistic model }} & 0.5357634 & -733.55738 & 1479.1148 & 1494.1799 \\
\hline & & & & 0.9456330 & -49.67684 & 114.7031 & 130.9297 \\
\hline
\end{tabular}

TABLE II

Fit parameters and fit quality indicators for the amended and improved amended model $B$ discussed in the text. For the comparison purposes the fit indicators for the linear and logistic models are also presented. Values in bold are insignificant from the perspective of the fit quality.

\begin{tabular}{|c|c|c|c|c|c|c|c|c|c|}
\hline \multicolumn{10}{|c|}{ Model $B$} \\
\hline \multicolumn{4}{|c|}{ alpha } & \multicolumn{4}{|c|}{ beta } & \multicolumn{2}{|c|}{$y$} \\
\hline GDP & CBIR & NTR & Intercept & GDP & CBIR & NTR & Intercept & Price & Intercept \\
\hline-0.002849 & -0.2031 & 0.003375 & 3.968 & -0.2011 & 1.241 & -0.02543 & 19.89 & 0.00173 & -5.846 \\
\hline $\begin{array}{l}\text { fitting } \mathrm{q} \\
\text { linear } \mathrm{n} \\
\text { logistic } \\
\text { cusp } \mathrm{m}\end{array}$ & $\begin{array}{l}\text { ality } \\
\text { odel } \\
\text { lodel } \\
\text { del }\end{array}$ & $\begin{array}{l}0.15 \\
0.54 \\
0.91\end{array}$ & $\begin{array}{l}011 \\
764 \\
785\end{array}$ & $\begin{array}{r}\log 1 \\
-761.0 \\
-732.6 \\
-38 .\end{array}$ & $\begin{array}{l}\mathrm{k} \\
015 \\
533 \\
623\end{array}$ & $\begin{array}{r}A \\
153 \\
148 \\
97 .\end{array}$ & $\begin{array}{l}\mathrm{C} \\
0803 \\
2706 \\
245\end{array}$ & $\begin{array}{r}\mathrm{B} \\
154 \\
150 \\
12\end{array}$ & $\begin{array}{l}\mathrm{C} \\
635 \\
868 \\
521\end{array}$ \\
\hline \multicolumn{10}{|c|}{ Improved model $B$} \\
\hline \multicolumn{4}{|c|}{ alpha } & \multicolumn{4}{|c|}{ beta } & \multicolumn{2}{|c|}{$y$} \\
\hline GDP & CBIR & NTR & Intercept & GDP & CBIR & NTR & Intercept & Price & Intercept \\
\hline 0 & -0.2357 & 0 & 1.348 & 0 & 1.052 & -0.02871 & 0 & 0.001705 & -5.766 \\
\hline $\begin{array}{l}\text { fitting } \mathrm{q} \\
\text { linear } \mathrm{n} \\
\text { logistic } \\
\text { cusp } \mathrm{m}\end{array}$ & $\begin{array}{l}\text { ality } \\
\text { odel } \\
\text { odel } \\
\text { del }\end{array}$ & $\begin{array}{l}0.14 \\
0.37 \\
0.92\end{array}$ & $\begin{array}{r}280 \\
3489 \\
763\end{array}$ & $\begin{array}{r}\log 1 \\
-761.3 \\
-747.0 \\
-42.2\end{array}$ & $\begin{array}{l}\mathrm{k} \\
774 \\
530 \\
325\end{array}$ & $\begin{array}{r}A \\
1530 \\
1504 \\
96 .\end{array}$ & $\begin{array}{l}\mathrm{C} \\
7548 \\
5059 \\
649\end{array}$ & $\begin{array}{r}\mathrm{B} \\
1540 \\
1516 \\
111\end{array}$ & $\begin{array}{l}\mathrm{C} \\
7189 \\
6049 \\
516\end{array}$ \\
\hline
\end{tabular}

All the fit quality measures indicate that neither the linear model nor the logistic one is better than the catastrophe model. First of all, significant advantage of the catastrophe model over the rest is seen in $R^{2}$ values. Similar conclusion can be drawn taking into consideration other measures: logLik, AIC, and BIC. It is necessary to adopt the following rules: the higher value of logLik, the better the model's fit to the data, whereas a better fit is achieved at lower values of AIC and BIC. Model modification and setting the free term in the asymmetry index to zero (lower part of Table I) also has a minor influence 
on the fit quality — the difference between original model $A$ and its modified version is small.

In model $B$ (Table II), control variables were extended to include the number of transactions in the real property market. Unexpectedly, the GDP value became an insignificant fit parameter (in both the asymmetry and bifurcation indexes). The number of transactions was also significant for the bifurcation index only. In its modified version (lower part of Table II), model $B$ had a free term in the bifurcation index set to zero.

Despite the larger number of parameters (more flexible fitting), model $\mathrm{B}$ appeared to have lower $R^{2}$ value than model $A$, but the catastrophe model proved to be definitely better than the others nevertheless. In turn, the remaining fit quality measures show the supremacy of model $B$ over both versions of model $A$.

Analysis of significance of canonical variables in model $B$ does not allow to draw any clear conclusion: some of the indicators $\left(R^{2}, \mathrm{AIC}, \mathrm{BIC}\right)$ indicate better fit in case of the catastrophe model, however the logLik value indicates the opposite. In the modified version of this model, the advantage of the catastrophe model over the logistic and the linear models increases.

\section{Conclusions}

Analyzing the results of studies it seems that periods of sudden price changes represent an intrinsic characteristic of the real property market in that sense that they represent turning points in the search for the equilibrium state of the system. Better fit measures of the cusp model with respect to the linear and logistic models suggest that the temporal hypothesis about sudden changes of real estate prices through discontinuous steps can be accepted as a base for further analysis.

Deeper understanding of the real estate dynamics using the catastrophe theory might eventually be used for prediction of sudden price changes. To achieve that goal it is necessary to extend the research area into wider spatial and temporal margins, including international markets, and longer time perspective. It is also essential to include additional observables into the model, to verify their influence on the system state. Having such results it would be possible to develop robust indicators of a catastrophe in the real estate market. The analyzed system's current status and probable development scenario, which will take the system in the direction of structural instability or structural stability, can be illustrated with a graphic representation of the evolutionary path.

\section{References}

[1] J. Castro, H. Swain, Lectures Comput. Sci. 40, 388 (1976).

[2] A. Okniński, Catastrophe Theory in Chemistry, Państwowe Wydawnictwo Naukowe, Warszawa 1990 (in Polish).

[3] P.A.I. Hartelman, H. van der Maas, A. Ploeger, Psychonomic Bull. Rev. 9, 26 (2002).

[4] A. Clark, Europ. Phys. J. B 50, 659 (2006).

[5] J. Barunik, M. Vosvrda, J. Econom. Dynam. Control 33, 1824 (2009).

[6] T. Figlus, The research on the settlement development and morphogenesis of olenderskie villages in Poland, in: Basic research and application of geographical sciences, Ed. L. Kasprzak-Bogucki, Wydawnictwo Naukowe, Poznań 2009 (in Polish).

[7] J. Bladowski, Morfogenesis of buildings, http:// virtual-city.eu/publikacje (2010) (in Polish).

[8] A. Jakimowicz, Acta Phys. Pol. A 117, 640 (2010).

[9] L. Cobb, B. Watson, Math. Model. 1, 311 (1980).

[10] L. Cobb, Behavior. Science 26, 75 (1981).

[11] P.A.I. Hartelman, Ph.D. Thesis, Faculteit Psychologie, Universiteit van Amsterdam 1997.

[12] P.A.I. Hartelman, H.L.J. van der Maas Hartelman, P.C.M. Molenaar, Brit. J. Developmental Psychol. 16, 97 (1998).

[13] E.J. Wagenmakers, P.C.M. Molenaar, P.A.I. Hartelman, H.L.J. van der Maas, Physica D 211, 263 (2005).

[14] R. Grasman, H.L.J. van der Maas, E.J. Wagenmakers, J. Statist. Software 32, 1 (2009).

[15] K. Żelazowski, J. Pol. Real Estate Soc. 19, 3 (2011) (in Polish).

[16] R. Trojanek, Price fluctuations in the housing market, Wydawnictwo Akademii Ekonomicznej w Poznaniu, Poznań 2008 (in Polish). 